Research Article

\title{
A Study on the Dynamic Responses and Joints Clearance Optimization of the Planar Operating Mechanism of High-Voltage Circuit Breaker
}

\author{
Xiaofeng Li, ${ }^{1}$ Shijing Wu $\mathbb{C D}^{1}{ }^{1}$ Xiaoyong Li $\mathbb{D}^{1,2}$ Deng Zhao, ${ }^{1}$ and Qiaoquan $\mathrm{Li}^{1}$ \\ ${ }^{1}$ Hubei Key Laboratory of Waterjet Theory and New Technology, Wuhan University, Wuhan 430072, China \\ ${ }^{2}$ Suzhou Institute of Wuhan University, Wuhan University, Suzhou 215000, China \\ Correspondence should be addressed to Shijing Wu; 2011301390031@whu.edu.cn and Xiaoyong Li; cqulixiaoyong@163.com
}

Received 24 January 2019; Revised 27 March 2019; Accepted 11 April 2019; Published 2 May 2019

Academic Editor: Angelo Marcelo Tusset

Copyright (c) 2019 Xiaofeng Li et al. This is an open access article distributed under the Creative Commons Attribution License, which permits unrestricted use, distribution, and reproduction in any medium, provided the original work is properly cited.

\begin{abstract}
The operating mechanism is responsible for a large part of machinery faults of high-voltage circuit breaker, which plays a crucial role in the reliability of the power grid. It consists of multiple flexible links connected by revolute joints with inevitable clearances between adjacent links. Flexible links and joints clearance have negative effects on the performance of the whole mechanical system. In this study, the dynamic simulation model of the multiple links articulated operating mechanism is built, and its validity is testified by operation experiment. Then, numerical simulation investigations are implemented to analyze the effects of flexible links and joints clearance on the dynamic responses of the operating mechanism. The results demonstrate that both flexible links and joints clearance exert associated negative effects on the mechanism performance. Furthermore, aimed at improving the dynamic characteristics of the operating mechanism, the joints clearance sizes are selected as design variables, and correlated optimization works are performed. After that, the acceleration and reaction force curves of the mechanical system become smoother, and the amplitudes of the two curves are reduced distinctly.
\end{abstract}

\section{Introduction}

High-voltage circuit breaker (HVCB) is the most important protection and control apparatus in the power grid. Generally speaking, the most important function of HVCB is automatically cutting the fault electric circuits off power transmission line. Due to the advantages of low cost and easy maintenance, multiple links articulated operating mechanism is widely applied in HVCB [1]. However, according to the statistics data, the fault caused by the operating mechanism contributes to a large part of HVCB service failures with $63.8 \%$ [2]. On the other hand, increased performances are expected for HVCB with the development of technologies in the power grid. Thus, it is meaningful to investigate the dynamic responses and optimization of the operating mechanism.

In multiple links articulated operating mechanism, joints clearance is unavoidable due primarily to the design, manufacturing process, and wear effects [3]. Clearance area in the joint can be simplified as a small unconstrained space, where the positions of shaft and bushing are random to a certain degree. Alternating separation and contact between the shaft and bushing frequently occur in joints clearance space. Impact reaction forces and vibration outputs of the mechanism are stimulated by these motion mode transformations in clearance joints, which eventually cause chaos in dynamic responses of mechanism [4-7].

Over the past few decades, many works on the joints clearance have been implemented. Continuous mode model, two-mode model, and three-mode model were put forward successively to describe the transitions between the noncontact and contact situation in clearance revolute joints [8-10]. As for the quantitative of impact forces, Flores et al. [11-13] had valuable contribution to different mathematical models for impact forces calculation in joints clearance. Besides, dry and viscose frictions at the classic joint connection, different joint models and clearance values, and different working conditions of mechanical system having 
rigid and flexible links were investigated [14-16]. It was found that the mechanism's dynamic responses are quite sensitive to the clearance size and number and can be degraded by joints clearance clearly. According to the abovementioned research studies, joints clearance generally leads to huge impact forces and strong vibration and eventually deteriorates the performance of the mechanical system. Bai and Zhao $[17,18]$ comparatively studied different mathematical calculation models for impact forces. A hybrid contact model with variable stiffness coefficient and damping coefficient is proposed for the impact forces calculation between the shaft and bushing in the clearance joints.

Furthermore, for the case of the operating mechanism, it contains multiple flexible links and features high speed. Chen et al. [19] pointed out that there might be deformation and vibration in flexible links under the high-speed condition, and the dynamic responses of the whole mechanical system are more sensitive to component's flexibility. Thus, the dynamic responses of the operating mechanism are likewise affected greatly by flexible links. Erkaya and Uzmay [20] conducted a comparative study on the dynamic responses of the slider-crank mechanism with and without flexural pivot. In addition, an experimental test rig was built, and more flexible flexural pivots were added to compliant mechanism between connecting rod and piston links [21]. A suspension effect of flexible elements on mechanism was discovered by and Li et al. [22], which makes the dynamic output of the slider-crank mechanism with flexural pivot more stable. Besides, a spring is attached between the coupler and the follower of a four-bar mechanism with clearance joint by Ahmedalbashir et al. [23]. His simulation results revealed that the separation between the shaft and bushing in joint is prevented by the preload of spring. Therefore, the impact forces and vibration amplitude caused by joints clearance decreased to some degree. Meanwhile, through experiment investigations and FFT analysis, Wang et al. [24] noticed that the maximum frequency of vibration responses was not affected by material parameters. The connecting shaft in clearance joint with lower Young's modulus can bring about lower nonlinear contact stiffness, which eventually causes delay effects to the mechanical system. Furthermore, rigid-flexible solar array system with clearance joints is modeled by Li et al. [25]; more complicated coupling effects between joints clearance and panel flexibility on the dynamic responses of the mechanical system are numerically analyzed. Salahshoor et al. [26] made further contributions to the comprehensive effects of joints clearance and flexible elements upon the vibration behaviors of the slider-crank mechanism. More stable output vibration with decreased magnitude can be obtained when element stiffness varied in a specific range. It suggests that the component flexibility cannot be ignored for the dynamic response analysis in articulated multibody mechanisms. In addition to planar joint, the dynamic responses of mechanical systems with three-dimensional clearance joints also attracted much attention in recent years [27-29].

As a key transmission mechanism, the dynamic responses of the operating mechanism directly link to the operation quality of HVCB. In the past decades, lots of the machinery faults of HVCB caused by the operating mechanism have been detected $[30,31]$. Therefore, related investigations about the joints clearance and flexible linkinduced effects on the operating mechanism are crucial for the stable operation of HVCB. However, the crank-slider and four-bar mechanisms with flexible elements and clearance joints are sorted mainly as the study objects in most of the current studies. Relative works on multiple links articulated operating mechanism are absent. For this situation, the dynamic responses of the articulated operating mechanism with multiple flexible components and clearance revolute joints are considered in this study. Different from the crank-slider and four-bar linkage mechanism, its operation is a nonperiodic transient impact process, and eight joint clearances are contained which make the dynamic responses present more complicated. At first, the dynamic simulation model of the operating mechanism was built under MSC ADAMS and verified through experiment. Dynamic simulation cases under different conditions with and without flexible links, as well as joints clearance, were proceeded sequentially. Then, the effects of multiple joints clearance and flexible links on the dynamic response of the operating mechanism were performed through contrastive analysis. Further optimization based on joints clearance size was carried out in ADAMS/ Insight to improve the dynamic characteristics of the mechanism.

This paper is organized as follows: Section 1 introduces the structure of the operating mechanism of HVCB. Dynamic simulation model establishment and its experiment investigation are outlined in Section 2. Obtained simulation results of different scenarios are described in Section 3. Further optimization is described in Section 4. The final conclusion of the whole study is given in Section 5 .

\section{Simulation Model of Operating Mechanism}

2.1. Operating Mechanism of HVCB. A typical multiple links articulated operating mechanism is illustrated in Figure 1. The purple arrow represents the movement direction of the mechanism, and the red arrow denotes the resistance. The whole mechanism contains multiple links and two types of main motion pairs: two translation pairs (I and J in Figure 1) and eight rotation motion pairs $(\mathrm{A}, \mathrm{B}, \mathrm{C}, \mathrm{D}, \mathrm{E}, \mathrm{F}, \mathrm{G}$, and $\mathrm{H}$ in Figure 1). Multiple links are connected through revolute joints to realize the motion and force transmission. Except for negative effects, joints clearance is also indispensable for the mobility between adjacent links. Acting as execute component for HVCB, moving contact is constrained by translation motion and driven by the operating mechanism. The initial parameters of the mechanism such as mass and inertia parameters and geometric parameters are listed in Table 1.

During the operation, the upper link inputs the external mechanical energy and rotates clockwise, and the moving contact moves downward to disconnect electric circuit. Since high-temperature and high-pressure arc is generated in this process, the operation must obey the following rules: 


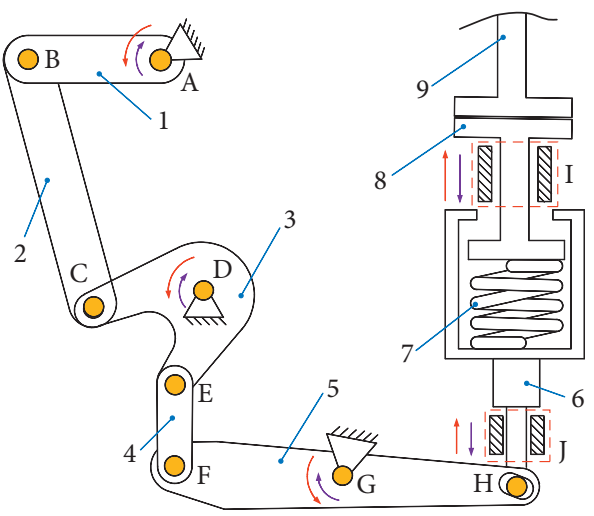

FIGURE 1: The schematic diagram of the operating mechanism. 1: upper link; 2: middle link; 3: upper arm; 4: down link; 5: down arm; 6: insulating link; 7: buffer spring; 8: moving contact; and 9: static contact.

TABLE 1: Inertia and geometric parameters of the mechanical system.

\begin{tabular}{lccc}
\hline Parameter & Length $(\mathrm{m})$ & Mass $(\mathrm{kg})$ & Centroid $(\mathrm{m})$ \\
\hline Upper link & 0.082 & 0.0748 & 0.029 (to A) \\
Middle link & 0.116 & 0.215 & 0.045 (to B) \\
Upper arm & 0.071 & 0.227 & 0.015 (to D) \\
Down link & 0.078 & 0.117 & 0.027 (to E) \\
Down arm & 0.208 & 0.542 & 0.014 (to F) \\
Insulating link & $\backslash$ & 0.973 & $\backslash$ \\
Moving contact & & 2.270 & $\backslash$ \\
\hline
\end{tabular}

the displacement of moving contact is equal or greater than $12 \mathrm{~mm}$ and the operation time is limited within $0.03 \mathrm{~s}$.

2.2. Model of Revolute Joints with Clearance. The theoretical studies based on nonlinear dynamic equations are popular in simple mechanical system. Nevertheless, for the case of multiple links articulated operating mechanism, flexible links and joints clearance are contained in the mechanical system. The feasibility of this methodology is limited by the complexity of the nonlinear dynamic equation. On the other hand, experimental studies can obtain more accurate results without considering complicated dynamic equations. It is quite expensive and time-consuming to carry out all experimental studies. Furthermore, the experimental results can only reflect the integrated effects of flexible links and joints clearance. Comprehensively, the dynamic simulation model is built under MSC ADAMS to analyze the dynamic responses of the operating mechanism in this paper. Based on the contact theory, the revolute joint model with clearance is established and embedded in the dynamic simulation model. Through substructure modal synthesis, flexible links are further imported into the model. Comparative simulation investigations in different operation conditions are carried out to progressively study the effects of flexible links and joints clearance on the dynamic responses of the mechanical system.

In the presence of joints clearance, two additional freedoms between shaft and bushing are introduced. Thus, the positions of shaft and bushing are uncertain in joints clearance to some extent during the operation. As shown in Figure 2, there are three types of motion modes: separation, contact, and penetration. Meanwhile, impact forces are produced accompanying the contact and penetration motion modes in clearance joints.

Through the calculation of penetration depth $\delta$ in the contact area, the three motion states between the shaft and bushing in Figure 2 can be judged: $\delta>0$ relates to separation state; $\delta=0$ corresponds to start of contact state; $\delta<0$ for penetration state.

$$
\left\{\begin{array}{l}
c=r_{\mathrm{b}}-r_{\mathrm{s}}, \\
\delta=\left|e_{i j}\right|-c,
\end{array}\right.
$$

where $r_{\mathrm{b}}$ and $r_{\mathrm{s}}$ represent the diameter of bushing and shaft, respectively, $c$ signifies the quantification of radius difference between shaft radii and bushing radii, and $e_{i j}$ denotes the deviation vector from bushing center $o_{\mathrm{b}}$ to shaft center $o_{\mathrm{s}}$. The impact forces between shaft and bushing can damage the operation accuracy and reduce the service life of the whole mechanical system. The impact forces consist of normal force $F_{\mathrm{n}}$ and tangential friction force $F_{\mathrm{t}}$.

$$
\begin{cases}F=0, & \delta<0, \\ F=F_{\mathrm{n}}+F_{\mathrm{t}}, & \delta \geq 0 .\end{cases}
$$

In contact and penetration motion modes, tangential friction force $F_{\mathrm{t}}$ is calculated by Coulomb's friction law. The contact force calculation in our paper is based on LankaraniNikravesh contact force model using the Hertz law together with a nonlinear viscoelastic element as [32]

$$
\left\{\begin{array}{l}
F_{\mathrm{t}}=F_{\mathrm{n}} \mu, \\
F_{\mathrm{n}}=K \delta^{\mathrm{n}}+D v,
\end{array}\right.
$$

where $\mu$ is the coefficient of friction, $n$ and $v$ represent the normal force index and the contact velocity, respectively, $K$ and $D$ denote the stiffness coefficient and damping coefficient separately, and $K$ depends on the structure and material properties of the contact area. The calculation formulas are expressed as follows:

$$
\begin{aligned}
& K=\frac{4\left(E_{\mathrm{b}} E_{\mathrm{s}}\right)}{3\left(E_{\mathrm{b}}\left(1-\varepsilon_{\mathrm{s}}^{2}\right)+E_{\mathrm{s}}\left(1-\varepsilon_{\mathrm{b}}^{2}\right)\right)}\left(\frac{r_{\mathrm{b}} r_{\mathrm{s}}}{r_{\mathrm{b}}-r_{\mathrm{s}}}\right)^{1 / 2}, \\
& D=\frac{3\left(1-c_{\mathrm{e}}^{2}\right) K \delta^{\mathrm{n}}}{4 v_{0}},
\end{aligned}
$$

where $\varepsilon$ and $E$ are Poisson's ratio and Young's modulus of each contact body, respectively, $v_{0}$ denotes the initial contact velocity, and $c_{\mathrm{e}}$ is the restitution coefficient. During our numerical simulation, Lankarani-Nikravesh force calculation model is written in $\mathrm{C}$ language and then embedded to our simulation model by dynamic link library method.

2.3. Simulation Model of Operating Mechanism. In the highspeed operation, the effects of flexible links on the dynamic response of the operating mechanism cannot be ignored. Through the substructure modal synthesis method, the whole 


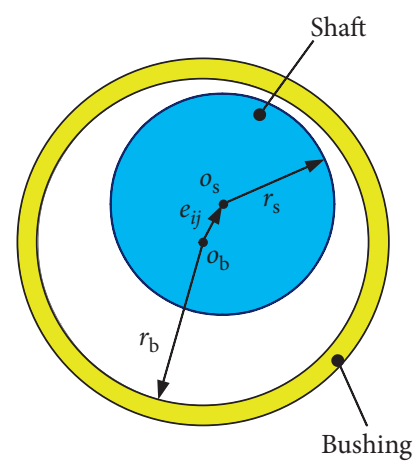

(a)

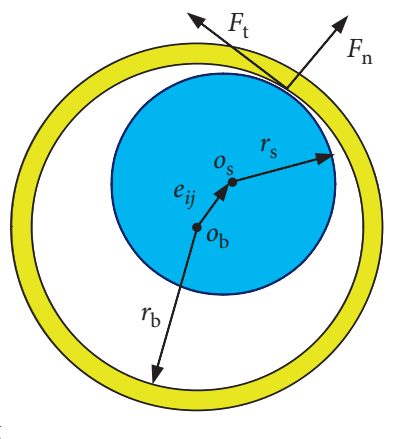

(b)

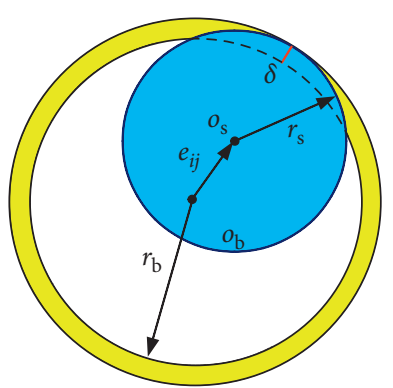

(c)

Figure 2: Position between shaft and bushing. (a) Separation. (b) Contact. (c) Penetration.

complex operating mechanism can be divided into several substructures. Then the detailed modal analysis can be complied easily towards on the substructure that contains concerned flexible links to obtain the model data. Combining the modal data of these flexible links with the other rigid components, the flexible-rigid coupled dynamic simulation model of the whole operating mechanism can be set up.

As shown in Figure 3, the three-dimensional geometric model of the operating mechanism is first set up under UG modeling software and is brought into MSC ADAMS to establish the purely rigid simulation model. The key point of substructure modal synthesis method in this study is gaining the modal data of the flexible links. For this aim, the concerned flexible links of operating mechanism: upper link, middle link, upper arm, down link, and down arm are imported into finite analysis software ABAQUS. Meanwhile, 8 node hexahedron linear non-coordinated elements are applied to mesh these flexible components. The top 20 order modal data of these flexible links are extracted to form the modal neutral files. Obtained neutral files are further imported into the purely rigid model to replace their rigid counterparts. Combined with the other rigid components, buffer spring, moving contact, and static contact, the flexible-rigid coupled dynamic simulation model of the whole operating mechanism is established. Furthermore, the clearance revolute joint model mentioned above in Section 2.1 is embedded into the flexible-rigid coupled simulation model afterward. Last but not the least, total step number is 600 , total simulation time is $0.03 \mathrm{~s}$, tolerance error is set $1 \mathrm{e}^{-4}$, and integrator scheme with high-resolution accuracy called SI2 is adopted in our simulation model.

\section{Operation Experiment}

Operation experiment usually offers a good technique to obtain more accurate results. However, for the operating mechanism in this paper, it consists of multiple flexible links and clearance revolute joints. Multiple parameters need to be measured to evaluate the dynamic response of the mechanical system. The high cost and time-consuming features are some of the disadvantages for the complete experimental study. Particularly, the experimental results are affected by both flexible links and joints clearance; further deep analysis is hard to perform. Numerical simulation study offers an alternative solution that can overcome these deficiencies. Thus, the dynamic simulation model of the operating mechanism is constituted in the literature. To ensure the accuracy of simulation results, the validity of the simulation model needs to be testified through operation experiment before further simulation works. For that purpose, an operation experiment rig is set up in Figure 4.

The sensor measuring probe is connected with the moving contact of the mechanism to measure its displacement and velocity responses. For comparison, the same kinematic curves are also extracted from the dynamic simulation model with clearance joints in seventh level assembly tolerance. Obtained comparative results are given in Figure 5.

As shown in Figure 5, both the kinematic curves from experiment and simulation are not smooth, especially for the velocity curves. The fluctuations mean impact forces and vibration in the mechanical system, which could cause accuracy loss and reduced service life in HVCB. Beyond that, increasingly huge noise and vibration were found at the experimental site after multiple operation experiments. Disassembling the operating mechanism, accumulated deformations were detected in some flexible links, as well as wear between the shaft and bushing. All of these phenomena suggest that the flexible links and the joints clearance are the main reason for the deterioration of the operating mechanism. Compared with the simulation results, the kinematic curves of the simulation model are smoother. It is because the real external mechanical energy in the experiment is not completely stable, which also contributes to the nonlinearities of the dynamic responses. Furthermore, as demonstrated in Figure 4, the clearance size of several joints might be beyond the desired value on account of the wear effects. From the standpoint of the main curve trend, the simulation results have high consistency in the operation experiment data. Therefore, the effectiveness of the established dynamic simulation model is verified.

\section{Simulation Results and Analysis}

4.1. Ideal External Mechanical Energy. According to the analysis of Section 3, all the unstable external mechanical 


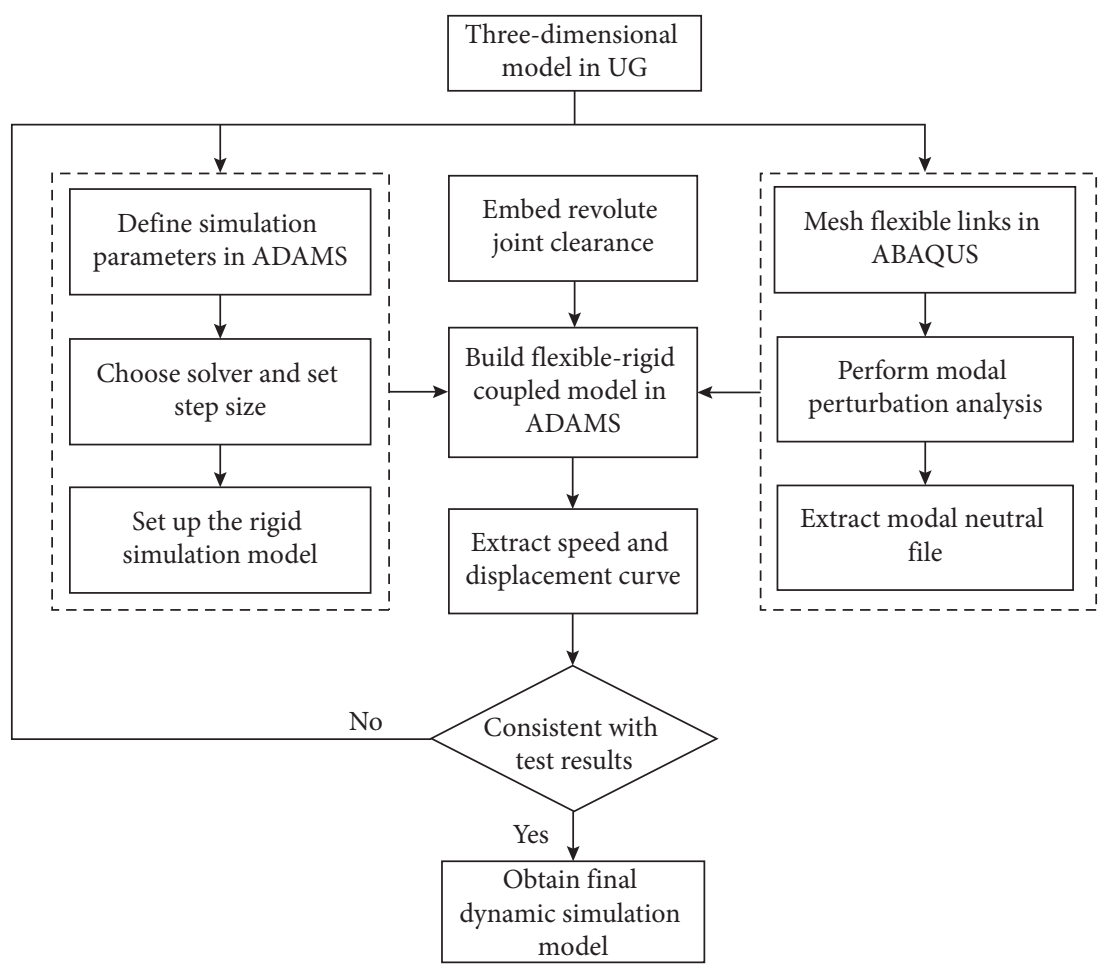

FIGURE 3: The modeling process of the operating mechanism.
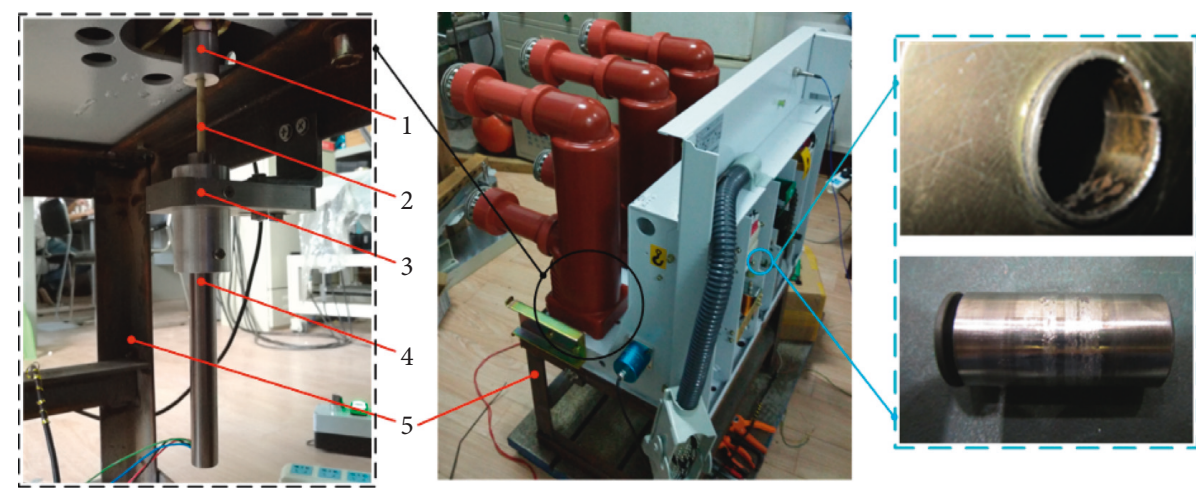

Figure 4: The operation experiment. 1: sleeve; 2: measuring probe; 3: auxiliary component; 4: sensor; and 5: bracket.

energy, the flexible links, and the joints clearance can make the mechanical system perform worse. Since these nonlinearities arising from unstable external mechanical energy are beyond the research in this paper, an ideal driven function is constituted as the external mechanical energy to actuate the operating mechanism. Then, the link flexibility and the joints clearance are introduced into the dynamic simulation model successively for further investigations. To eliminate the negative effects of the external mechanical energy on the dynamic responses of the mechanism, an ideal driven function is constructed as the actuation for the operating mechanism:

$$
x= \begin{cases}-19\left(\frac{t}{0.03}\right)^{2}\left(3-\frac{2 t}{0.03}\right), & 0 \leq t<0.03, \\ -19, & t \geq 0.03,\end{cases}
$$

where $x$ represents the displacement and $t$ denotes the operation time. Employing equation (5) as the angular displacement of the upper link, correlated simulation parameters under the ideal external mechanical energy are listed in Table 2.

Under the ideal driven function, corresponding kinematic outputs from the purely rigid dynamic simulation model without considering the element flexibility and joints clearance are given in Figure 6. It can be observed that the obtained kinematic curves of the rigid model are smooth during the operation. Thus, the proposed driven function is stable and has no negative effects on the dynamic responses of the mechanical system. Afterward, the flexible links and joints clearance can be introduced into the simulation model to investigate their effects on the operating mechanism. 


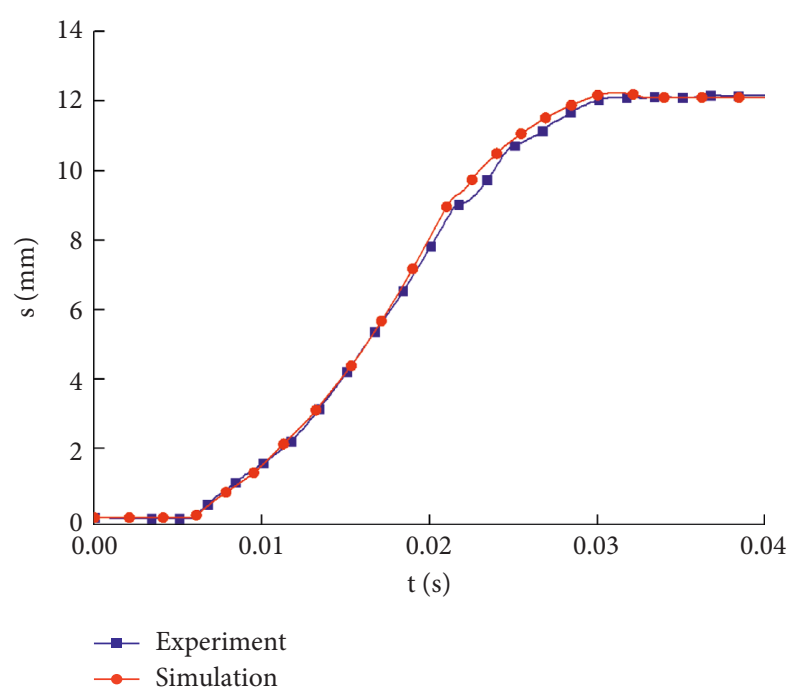

(a)

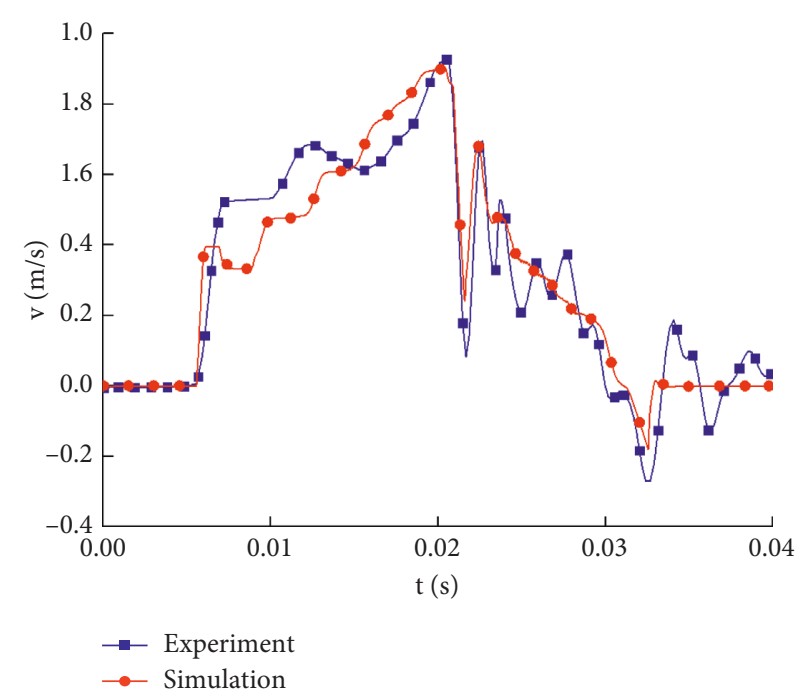

(b)

Figure 5: Displacement and velocity curves of the moving contact. (a) Displacement. (b) Velocity.

TABle 2: Dynamic simulation parameters.

\begin{tabular}{lc}
\hline Parameter name & Value \\
\hline$r_{\mathrm{s}}, r_{\mathrm{b}}(\mathrm{mm})$ & 6 \\
$v_{0}(\mathrm{~mm} / \mathrm{s})$ & 0 \\
$n$ & 1.5 \\
$c_{\mathrm{e}}$ & 0.9 \\
$\mu$ & 0.1 \\
$E_{\mathrm{s}}, E_{\mathrm{b}}(\mathrm{GPa})$ & 207 \\
$\varepsilon_{\mathrm{b}}, \varepsilon_{\mathrm{s}}$ & 0.3 \\
\hline
\end{tabular}

4.2. Simulation Results with Link's Flexibility. In order to evaluate the effects of flexible links on the dynamics behaviors, the joints are assumed to be ideal, whose clearance size is taken as zero. As the final execute component, the dynamic responses of moving contact reflect the performance of high-voltage circuit breaker. Hence, the displacement, speed, and acceleration of the moving contact are extracted from the dynamic simulation model for the case study. At the same time, the reaction force of revolute joint $\mathrm{H}$ is also considered because it is the closest revolute joint to the moving contact. The comparative results of the dynamic simulation model with and without considering the link's flexibility are given in Figure 6.

From Figure 6(a), it can be seen that the displacement curves of the two simulation models coincide well. It indicates that stable and consistent displacement output can be obtained with the ideal external mechanical energy input, regardless of flexible links. Therefore, the deformation in flexible links is negligible to the displacement output of the whole mechanism. By contrast, the speed, acceleration, and the reaction force curve from the two simulation models are significantly different. For detailed comparative analysis, these dynamic response curves are divided into five stages. In the first stage $\mathrm{O}-\mathrm{A}$, the speed, acceleration, and reaction force curves of the coupled model present obvious fluctuations. It is because that initial elastic deformation occurs in flexible links, and some kinetic energy is transformed into elastic potential energy stored in flexible links. Stability of the established ideal external mechanical energy is damaged by the energy transformation and storage process. Thus, fluctuations eventually appear in the dynamic response curves of the coupled model compared with the purely rigid model in this operation stage. $10 \mathrm{~ms}$ later, the operation enters into the second stage A-B, and the energy storage capacities of flexible links tend to be saturated. Under the circumstances, both the dynamic response curves of the two models are smooth and coincident at this stage. The moving contact of the rigid model reaches its speed peak at point $\mathrm{B}$, after which it started to decelerate. It is worth mentioning that due to the free-flight mode, the reaction force disappears at point $B$. For the coupled simulation model, owing to the partial mechanical energy loss caused by the energy transformation in the stage $\mathrm{O}-\mathrm{A}$, the moving contact needs more time to reach its peak velocity. Thus, it keeps accelerating until point $\mathrm{C}$ and then starts to decelerate and steps to the next stage C-D. Affected by the deceleration of the moving contact, the elastic potential energy in flexible links releases. Consequently, the speed, acceleration, and reaction force curves of the coupled simulation model fluctuate and deviate from that of the rigid simulation result again. In the final stage D-E, fluctuations of the moving contact are getting weaker because the stored elastic potential energy is depleted. The dynamic response curves of two simulation models end to coincide eventually. It can be found that flexible links can lead to fluctuations in the dynamic responses and a delay in peak velocity of the operating mechanism.

4.3. Simulation Results with Clearance Joints. There are eight clearance revolute joints (joint A, B, C, D, E, F, G, and H in Figure 1) in the operating mechanism of HVCB. In the joints clearance, separation and contact between shaft and bushing repeatedly emerge during the operation, which is considered 


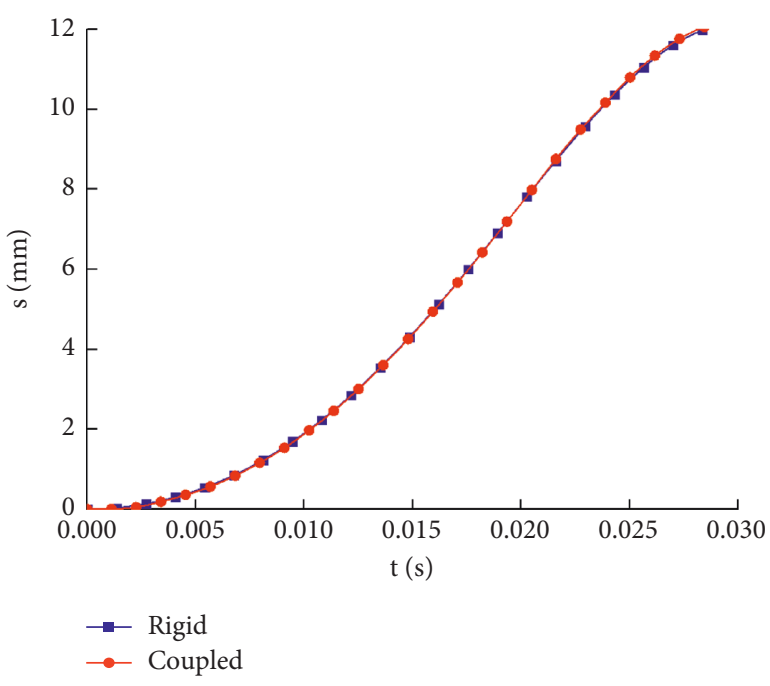

(a)

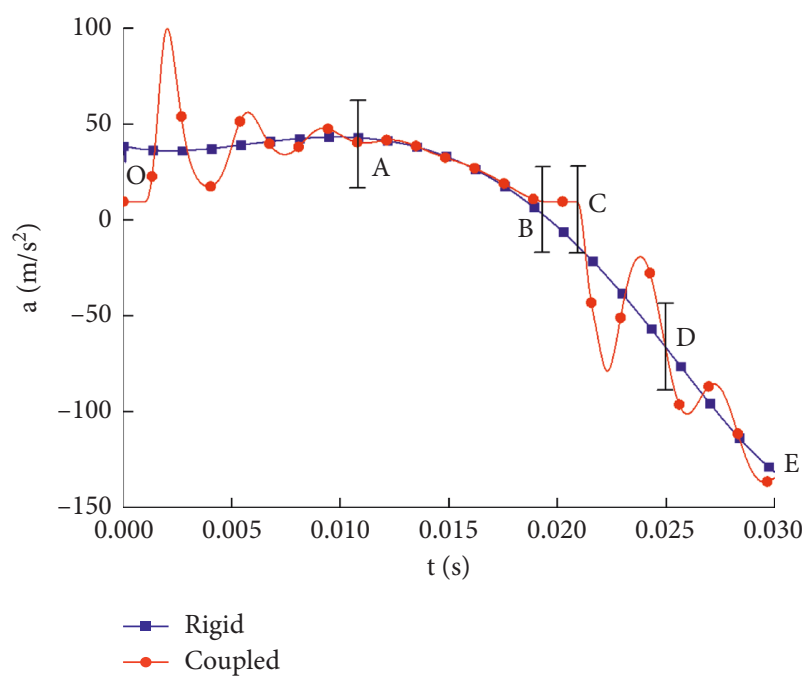

(c)

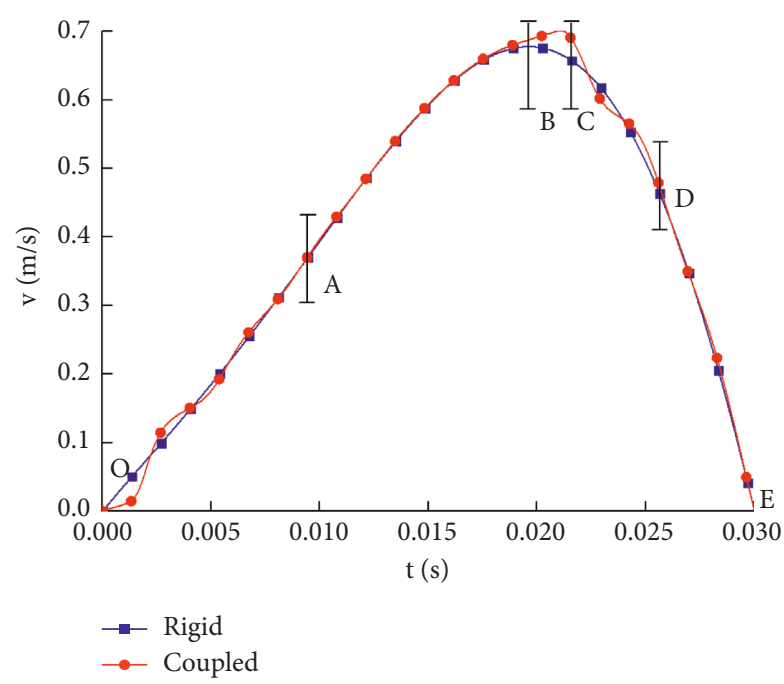

(b)

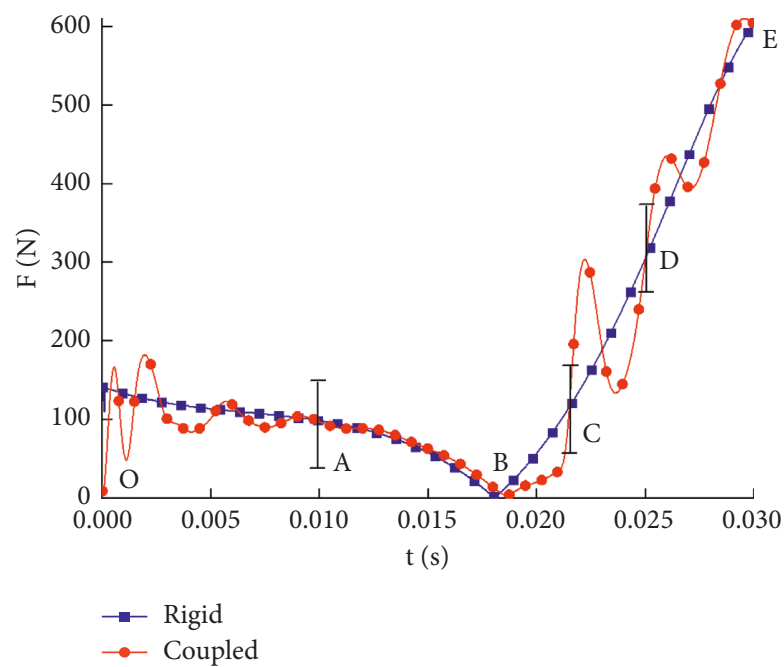

(d)

Figure 6: The simulation results of the rigid model and coupled model. (a) Displacement. (b) Velocity. (c) Acceleration. (d) Reaction force.

as the main source of impact forces in the articulated mechanical system. For the assessment of negative effects of joints clearance, joints clearances are further embedded into the flexible-rigid coupled dynamic simulation models based on the clearance revolute joints model in Section 2.2. For this aim, a mark point is set on the center position of shaft and bushing separately. In our numerical simulation process, the software measures the distance of the two-mark point every simulation step and then subtracts the value of the original diameter difference to obtain the penetration depth. It further differentiates this value for relative contact velocity. As for the initial clearance size, the seventh level assembly tolerance (IT7) is adopted in the simulation model for the initial case study of joints clearance. According to the relative manufacture standard, these joints clearance sizes in the simulation model are set as $30 \mu \mathrm{m}$. The simulation results are shown in Figure 7.

Similarly, both of the displacement curves from the rigid model and the coupled model are smooth and basically in coincidence with each other no matter whether the joints clearance are considered or not. Conversely, the velocity, acceleration, and reaction force curves obtained from the two simulation models show great differences. Compared with the case that only flexible links exist, dramatic oscillations are introduced by joint clearance which can also be found in Chen et al.'s research [33]. It means that joint clearance has greater effects on the dynamic responses of the mechanical system. Also, except for the displacement curve, the other dynamic response curves are divided into three main stages for the purpose of analyzing the effects of joints clearance. In the first stage O-A, obvious oscillations appear in the dynamic response curves of the two simulation models with joints clearance. In the occurrence of the initial motion state transition of the operating mechanism from static to motion, repeated separation and contact arise between the shaft and bushing in joints clearance. Accordingly, when the high impact forces are stimulated, the speed, acceleration, and reaction force curves of the moving contact 


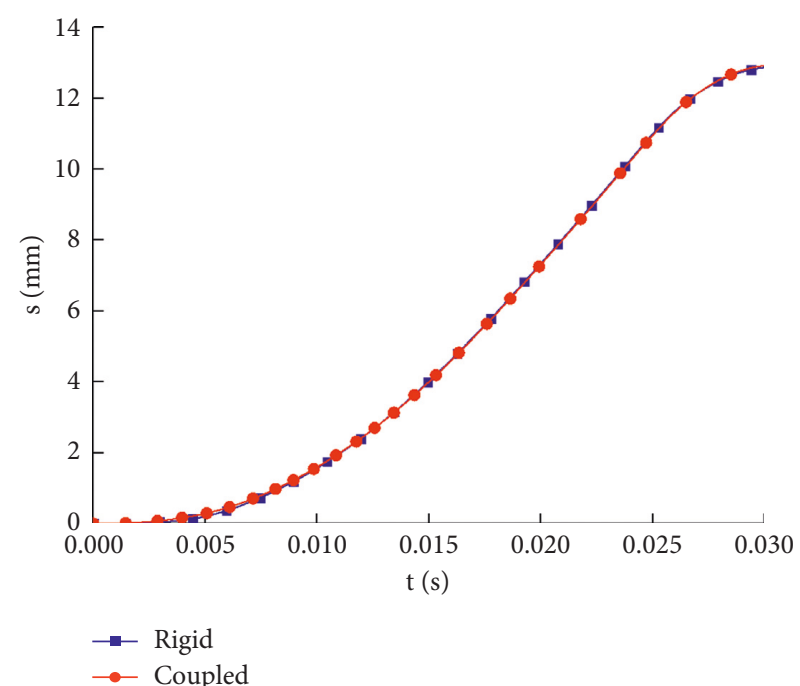

(a)

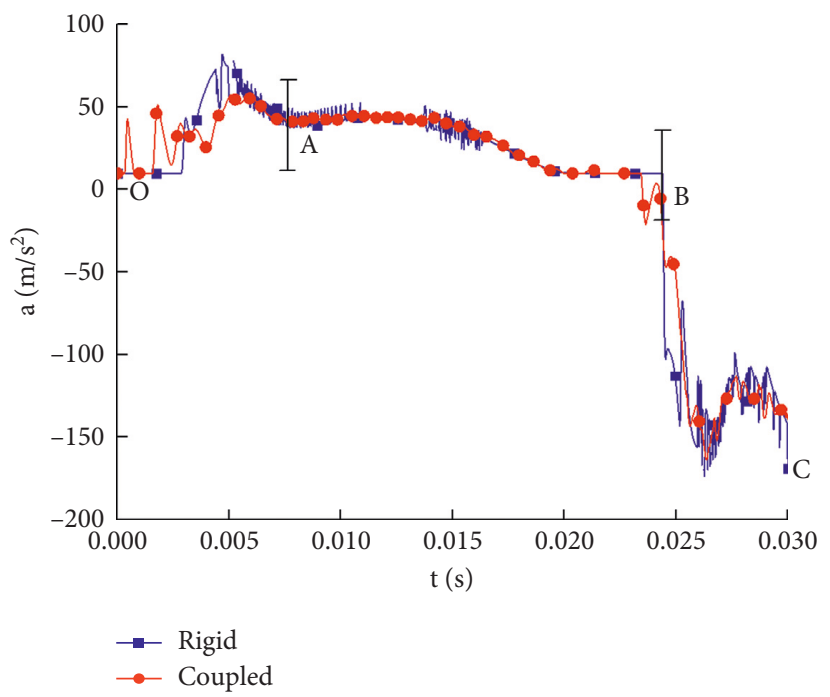

(c)

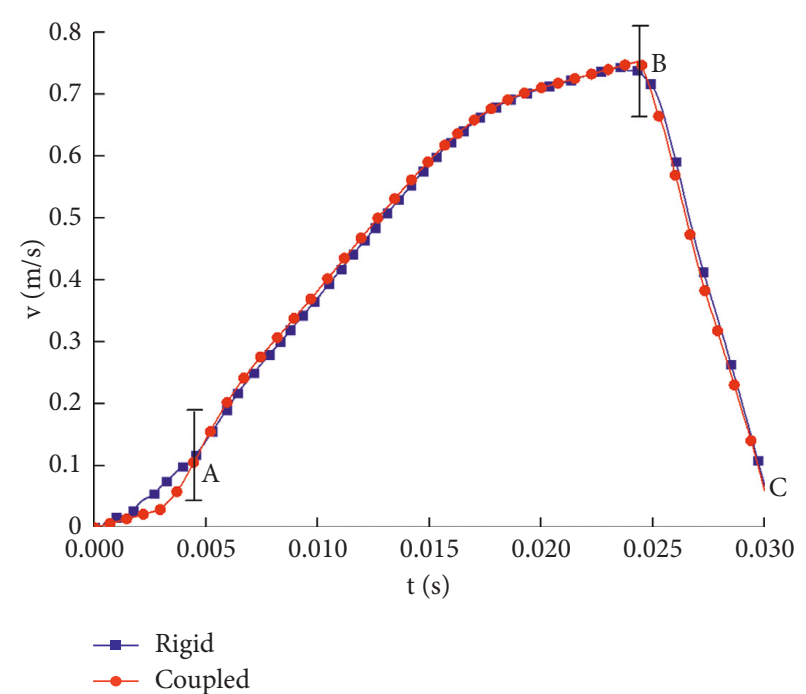

(b)

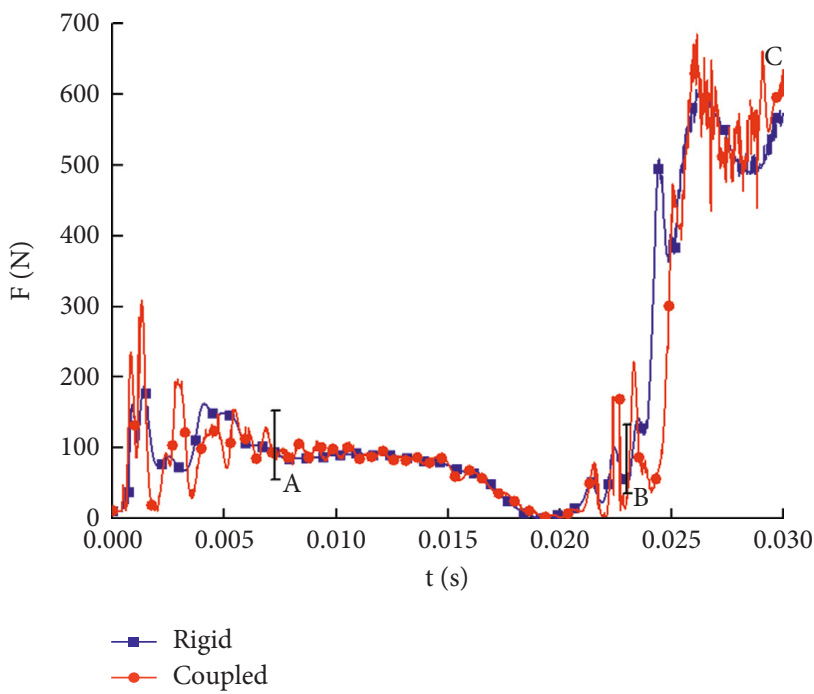

(d)

FIGURE 7: Comparative simulation results with clearance. (a) Displacement. (b) Velocity. (c) Acceleration. (d) Reaction force.

oscillate obviously. Afterward, the operating mechanism enters into a more stable motion stage A-B. Under the input of ideal external mechanical energy, dynamic response curves from the two simulation models are more steady and consistent. The speed curves of the two simulation models in Figure 7 almost reach peak point B simultaneously. It entails that the joints clearance can compensate some of the undesirable delay effects of flexible links. Then comes to the last operation stage $\mathrm{B}-\mathrm{C}$, when the moving contact starts to decelerate. The deceleration can cause the release of stored potential energy in flexible links, and further, give rise to the unstable separation and contact between the shaft and bushing in joints clearance. As a result of that, dynamic response curves start to oscillate again. Last but not the least, simultaneously affected by flexible links and clearance joints, more severe oscillations can be observed in the reaction force curve of the coupled model than that of the rigid model. Since these impact forces are the main reason for severe vibration, more oscillations in the acceleration stage of the coupled model should be in sight theoretically. Conversely, as shown in Figure 8(a), it is clear that the acceleration curve of the rigid model contains more high-frequency vibration. The explanation for this phenomenon is that the flexible links in the coupled model can absorb the energy of the vibration stimulated by impact forces and eventually weaken the vibration. Similar damping effects of flexible links on the dynamic responses are also found in Erkaya's studies [21]. Consequently, the flexible links can also decrease the negative effects of joints clearance.

4.4. Optimization of Revolute Joint. According to the experiment and simulation result, drastic oscillations arise in the dynamic responses of the operating mechanism with clearance joints in seventh level assembly tolerance. Oscillations in the acceleration curve mean the vibration in 
the moving contact, and the drastic oscillations in reaction force curve indicate that the operating mechanism is undertaking impact force. All these oscillations can deteriorate the mechanical stability and the performances of the whole system. Thus, relative dynamic optimization investigations are performed in the study under the experimental design and analysis module called ADAMS/ Insight. ADAMS/Insight is an additional module in ADAMS aimed for sensitivity and optimization analysis. During the iterative optimization, the RSM method is utilized for mathematical regression of multiple variable parameter simulation calculations. The objectives of optimization are to decrease the oscillations in acceleration and reaction force curve. Clearance size is considered as design variable because clearance joint exerts greater effects (compared with link's flexibility) on the dynamic response of the mechanical system, according to abovementioned simulation results. Compared with joints $\mathrm{A}, \mathrm{B}, \mathrm{C}$, and $\mathrm{D}$, joints E, F, G, and $\mathrm{H}$ are closer to moving contact. Thus, the clearance in joints E, F, G, and $\mathrm{H}$ might exert greater influence on the dynamic responses. Also, worse wear phenomena in these four joints are discovered while disassembling the operating mechanism in Section 4 . Therefore, constant eighth level assembly tolerance is adopted in joints A, B, C, and D. The joints E, F, G, and H are considered as optimization object, whose clearance size varies between sixth level (IT6) and eighth level (IT8). The diameter of the shaft in the operating mechanism is $12 \mathrm{~mm}$. The exact clearance size range of sixth level seventh and eighth level assembly tolerance level is $16-22 \mu \mathrm{m}$, $22-36 \mu \mathrm{m}$, and $36-54 \mu \mathrm{m}$, respectively. The final optimization objectives are to minimize the oscillations in acceleration and reaction force curves.

After multiple iterative simulations, the optimal clearance sizes are gained and listed in Table 3, and the optimal acceleration curve and reaction force curve are given in Figure 8.

It can be noticed that the acceleration and reaction force responses of the operating mechanism have been improved distinctly compared with the original results. For further analysis, both the acceleration and reaction force curves in Figure 8 are divided into 3 stages: O-F, F-G, and G-H. Firstly, taking the optimized acceleration curve as an instance, these oscillations of high frequency and small amplitude in stage $\mathrm{O}-\mathrm{F}$ are eliminated. Then, the operation enters into stable period F-G, during which the two curves tend to be consistent. Furthermore, the last stage G-F witnesses biggest optimization results. The optimized acceleration curve is smoother, and the amplitude decreases dramatically by $24.8 \%$. For the case of the reaction force curve, the whole reaction force curve is smoother than before. Meanwhile, after optimization, the peak value in the reaction force curve decreases by $18.4 \%$ and $26.1 \%$ during stage O-F and the last stage $\mathrm{G}-\mathrm{H}$, respectively. Also, the two curves tend to be closer under the more stable motion stage F-G. The optimization of the joints clearance size has improved the dynamic characteristics of the operating mechanism, which will enhance the performance of HVCB and the reliability of the power grid.
TABLE 3: The clearance size after optimization.

\begin{tabular}{lcccc}
\hline Revolute joint & E & F & G & H \\
\hline Clearance size $(\mu \mathrm{m})$ & 26.7 & 18.9 & 29.5 & 17.6 \\
Assembly tolerance level & IT7 & IT6 & IT7 & IT6 \\
\hline
\end{tabular}

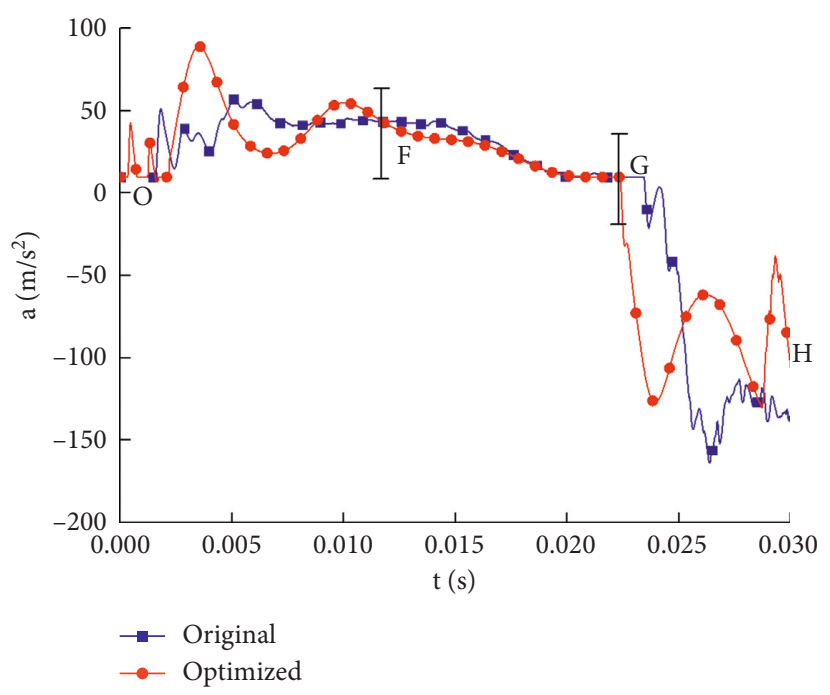

(a)

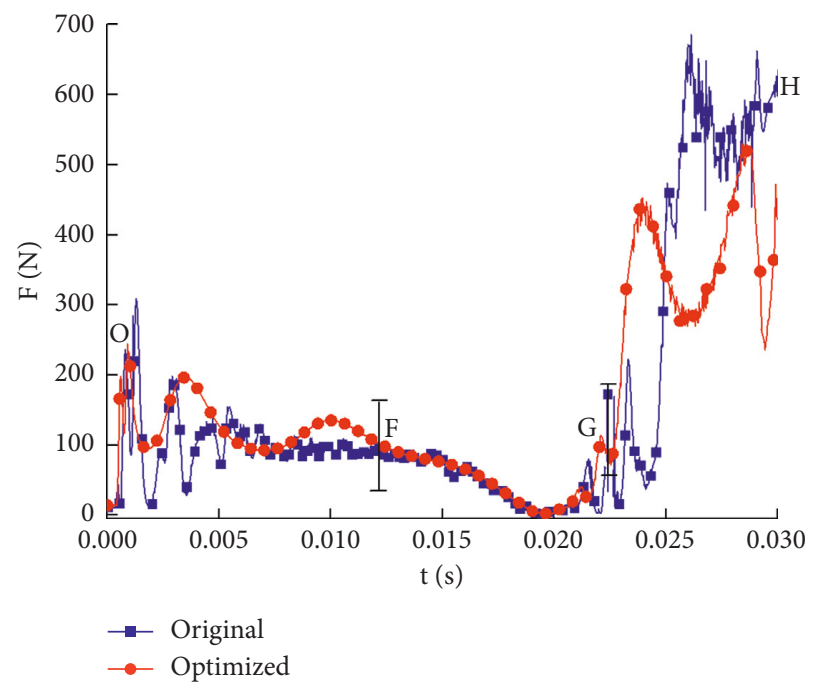

(b)

FIgURE 8: The optimization results of dynamic response. (a) Acceleration. (b) Reaction force.

In combination with Table 2, it can be concluded that smaller joints clearance is not always necessarily good for better dynamic responses when flexible links are considered. Larger joints clearance size can cause huger impact forces between shaft and bushing and further severe vibration in moving contact. Under the circumstances of larger joints clearance size, clearance joints play the dominant role in degrading the performance of the operating mechanism. In addition to negative effects, the joints clearance can also decrease the undesired effects of flexible links on the dynamic responses of the mechanical system. Thus, on the occasion of 
smaller joints clearance, the deteriorating effects of flexible links on the dynamic responses are fully manifested. Hence, the optimal joints clearance size combined with the operating mechanism needs to be determined comprehensively with the consideration of flexible links. Besides, more in-deep optimization investigations based on links' geometries/dimensions and material will be carried out in our further study.

\section{Conclusions}

Investigations of the dynamic responses and optimization of a classical multiple links articulated operating mechanism are implemented in the literature. For this aim, a flexible-rigid coupled dynamic simulation model of the operating mechanism is established and verified through relative operation experiment. Simulation investigations of the effects of the flexible links and joints clearance on the dynamic responses of the operating mechanism are performed. The simulation results indicate that both flexible links and joint clearance can exert negative effects on the dynamic response of the mechanical system. For the case of flexible links, the storage and release of the elastic potential energy of flexible links cause the fluctuation and subtle delay in the dynamic responses of the mechanical system. As for the clearance joint, it plays a dominant role in degrading the performance of the operating mechanism which could introduce dramatic oscillation in the dynamic responses. Besides, when both of the two factors are considered, associated effects on the dynamic responses are exerted by the flexible links and joints clearance. Moreover, joints clearance in eighth level assembly tolerance is adopted in joints $\mathrm{A}, \mathrm{B}, \mathrm{C}$, and $\mathrm{D}$, and further clearance size optimization is conducted based on the joints E, F, G, and H. After the clearance optimization, the amplitude of oscillation acceleration curve decreases by $24.8 \%$ in the whole operation stage and that of the reaction force decreases by $18.4 \%$ and $26.1 \%$ in the initial and end operation stage, respectively.

\section{Data Availability}

The experiment and simulation data in Figures 5, 6, 7, and 8 used to support the findings of this study are fully available from the corresponding authors upon request.

\section{Conflicts of Interest}

The authors declare that there are no conflicts of interest regarding the publication of this paper.

\section{Authors' Contributions}

Li Xiaofeng established the model, designed the experiments, and wrote the paper; Wu Shijing and Li Xiaoyong guided the system design and revised the manuscript; and Zhao Deng and Li Qiaoquan performed the experiments and analyzed the data.

\section{Acknowledgments}

The authors have received funds for covering the costs to publish in open access. This research was supported by the
National Science Foundation of China (no. 51705372) and Jiangsu Provincial Natural Science Foundation of China (no. BK20170411).

\section{References}

[1] X. Zhang, E. Gockenbach, Z. Liu, H. Chen, and L. Yang, "Reliability estimation of high voltage SF6 circuit breakers by statistical analysis on the basis of the field data," Electric Power Systems Research, vol. 103, pp. 105-113, 2013.

[2] F. G. Meng, S. J. Wu, F. Zhang, Z. L. Zhang, J. C. Hu, and X. Y. Li, "Modeling and simulation of flexible transmission mechanism with multiclearace joints for ultrahigh voltage circuit breakers," Shock and Vibration, vol. 2015, Article ID 392328, 17 pages, 2015.

[3] O. Muvengei, J. Kihiu, and B. Ikua, "Dynamic analysis of planar multi-body systems with LuGre friction at differently located revolute clearance joints," Multibody System Dynamics, vol. 28, no. 4, pp. 369-393, 2012.

[4] Z. F. Bai, X. Jiang, F. Li, J. J. Zhao, and Y. Zhao, "Reducing undesirable vibrations of planar linkage mechanism with joint clearance," Journal of Mechanical Science and Technology, vol. 32, no. 2, pp. 559-565, 2018.

[5] S. B. Farahan, M. R. Ghazavi, and S. Rahmanian, "Bifurcation in a planar four-bar mechanism with revolute clearance joint," Nonlinear Dynamics, vol. 87, no. 2, pp. 955-973, 2017.

[6] P. Varney and I. Green, "Nonlinear phenomena, bifurcations, and routes to chaos in an asymmetrically supported rotorstator contact system," Journal of Sound and Vibration, vol. 336, pp. 207-226, 2015.

[7] S. Rahmanian and M. R. Ghazavi, "Bifurcation in planar slider-crank mechanism with revolute clearance joint," Mechanism and Machine Theory, vol. 91, pp. 86-101, 2015.

[8] J. F. Deck and S. Dubowsky, "On the limitations of predictions of the dynamic response of machines with clearance connections," Journal of Mechanical Design, vol. 116, no. 3, pp. 833-841, 1994.

[9] S. Erkaya and I. Uzmay, "Investigation on effect of joints clearance on dynamics of four-bar mechanism," Nonlinear Dynamics, vol. 58, no. 1-2, pp. 179-198, 2009.

[10] A. A. Olyaei and M. R. Ghazavi, "Stabilizing slider-crank mechanism with clearance joints," Mechanism and Machine Theory, vol. 53, no. 7, pp. 17-29, 2012.

[11] P. Flores, "A parametric study on the dynamic response of planar multibody systems with multiple clearance joints," Nonlinear Dynamics, vol. 61, no. 4, pp. 633-653, 2010.

[12] P. Flores and H. M. Lankarani, "Spatial rigid-multibody systems with lubricated spherical clearance joints: modeling and simulation," Nonlinear Dynamics, vol. 60, no. 1-2, pp. 99-114, 2010.

[13] P. Flores, C. S. Koshy, H. M. Lankarani, J. Ambrósio, and J. C. P. Claro, "Numerical and experimental investigation on multibody systems with revolute clearance joints," Nonlinear Dynamics, vol. 65, no. 4, pp. 383-398, 2011.

[14] P. Flores, J. C. P. Ambrósio, J. C. Claro, and H. M. Lankarani, "Dynamics of multibody systems with spherical clearance joints," Journal of Computational and Nonlinear Dynamics, vol. 1, no. 3, pp. 240-247, 2006.

[15] Q. Tian, C. Liu, M. Machado, and P. Flores, "A new model for dry and lubricated cylindrical joints with clearance in spatial flexible multibody systems," Nonlinear Dynamics, vol. 64, no. 1-2, pp. 25-47, 2011.

[16] P. Flores, J. Ambrósio, J. C. P. Claro, and H. M. Lankarani, "Spatial revolute joints with clearances for dynamic analysis of 
multi-body systems," Proceedings of the Institution of $\mathrm{Me}$ chanical Engineers, Part K: Journal of Multi-Body Dynamics, vol. 220, no. 4, pp. 257-271, 2006.

[17] Z. F. Bai and Y. Zhao, "A hybrid contact force model of revolute joint with clearance for planar mechanical systems," International Journal of Non-Linear Mechanics, vol. 48, no. 48, pp. 15-36, 2013.

[18] Z. F. Bai and Y. Zhao, "Dynamic behaviour analysis of planar mechanical systems with clearance in revolute joints using a new hybrid contact force model," International Journal of Mechanical Sciences, vol. 54, no. 1, pp. 190-205, 2012.

[19] Y. Chen, Y. Sun, and C. Chen, "Dynamic analysis of a planar slider-crank mechanism with clearance for a high speed and heavy load press system," Mechanism and Machine Theory, vol. 98, pp. 81-100, 2016.

[20] S. Erkaya and I. Uzmay, "Modeling and simulation of joint clearance effects on mechanisms having rigid and flexible links," Journal of Mechanical Science and Technology, vol. 28, no. 8, pp. 2979-2986, 2014.

[21] S. Erkaya, "Experimental investigation of flexible connection and clearance joint effects on the vibration responses of mechanisms," Mechanism and Machine Theory, vol. 121, pp. 515-529, 2018.

[22] Y. Li, G. Chen, D. Sun, Y. Gao, and K. Wang, "Dynamic analysis and optimization design of a planar slider-crank mechanism with flexible components and two clearance joints," Mechanism and Machine Theory, vol. 99, pp. 37-57, 2016.

[23] M. Ahmedalbashir, L. Romdhane, and J. Lee, "Dynamics of a four-bar mechanism with clearance and springs-modeling and experimental analysis," Journal of Mechanical Science and Technology, vol. 31, no. 3, pp. 1023-1033, 2017.

[24] X. Wang, G. Liu, S. Ma, and R. Tong, "Study on dynamic responses of planar multibody systems with dry revolute clearance joint: numerical and experimental approaches," Journal of Sound and Vibration, vol. 438, pp. 116-138, 2019.

[25] Y. Li, C. Wang, and W. Huang, "Dynamics analysis of planar rigid-flexible coupling deployable solar array system with multiple revolute clearance joints," Mechanical Systems and Signal Processing, vol. 117, pp. 188-209, 2019.

[26] E. Salahshoor, S. Ebrahimi, and Y. Zhang, "Frequency analysis of a typical planar flexible multibody system with joint clearances," Mechanism and Machine Theory, vol. 126, pp. 429-456, 2018.

[27] F. J. Cavalieri and A. Cardona, "Non-smooth model of a frictionless and dry three-dimensional revolute joint with clearance for multibody system dynamics," Mechanism and Machine Theory, vol. 121, pp. 335-354, 2018.

[28] J. Ambrósio and J. Pombo, "A unified formulation for mechanical joints with and without clearances/bushings and/or stops in the framework of multibody systems," Multibody System Dynamics, vol. 42, no. 3, pp. 317-345, 2018.

[29] F. Marques, F. Isaac, N. Dourado, and P. Flores, "An enhanced formulation to model spatial revolute joints with radial and axial clearances," Mechanism and Machine Theory, vol. 116, pp. 123-144, 2017.

[30] E. A. L. Vianna, A. R. Abaide, L. N. Canha, and V. Miranda, "Substations SF6 circuit breakers: reliability evaluation based on equipment condition," Electric Power Systems Research, vol. 142, pp. 36-46, 2017.

[31] M. L. Liu, K. Q. Wang, L. J. Sunand, and J. J. Zhen, “Applying empirical mode decomposition (EMD) and entropy to diagnose circuit breaker faults," Optik, vol. 126, no. 20, pp. 2338-2342, 2015.
[32] J. Alves, N. Peixinho, M. T. Da Silva, P. Flores, and H. M. Lankarani, "A comparative study of the viscoelastic constitutive models for frictionless contact interfaces in solids," Mechanism and Machine Theory, vol. 85, pp. 172-188, 2015.

[33] X. L. Chen, S. Jiang, Y. Deng, and Q. Wang, "Nonlinear dynamics and analysis of a planar multilink complex mechanism with clearance," Shock and Vibration, vol. 2018, Article ID 6172676, 17 pages, 2018. 


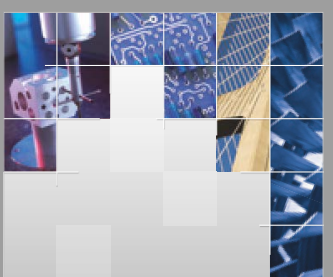

\section{Enfincering}
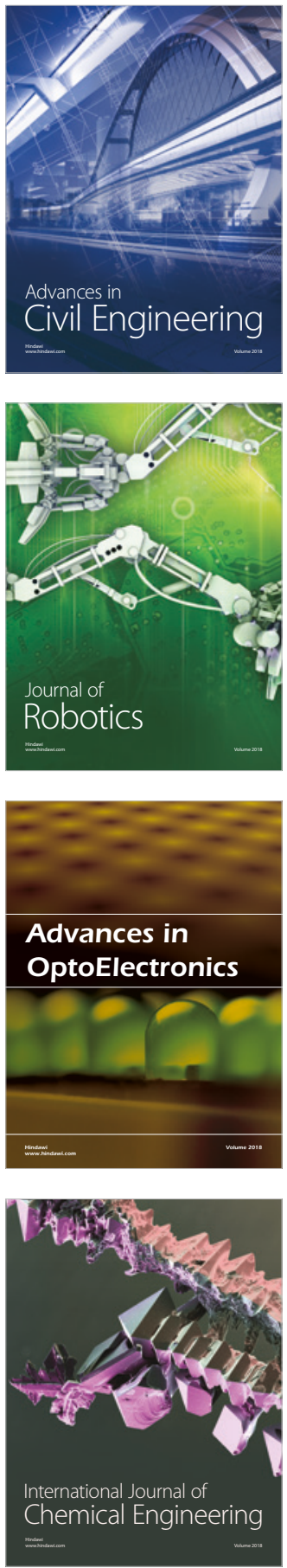

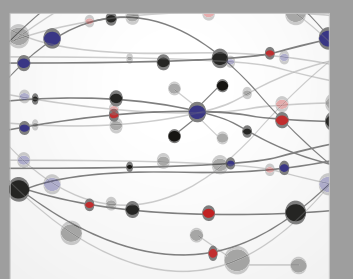

\section{Rotating \\ Machinery}

The Scientific World Journal

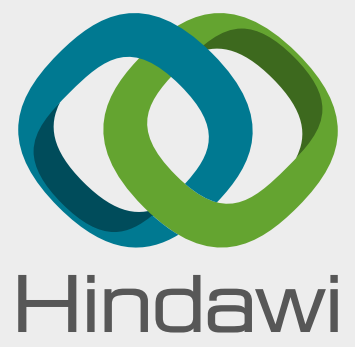

Submit your manuscripts at

www.hindawi.com
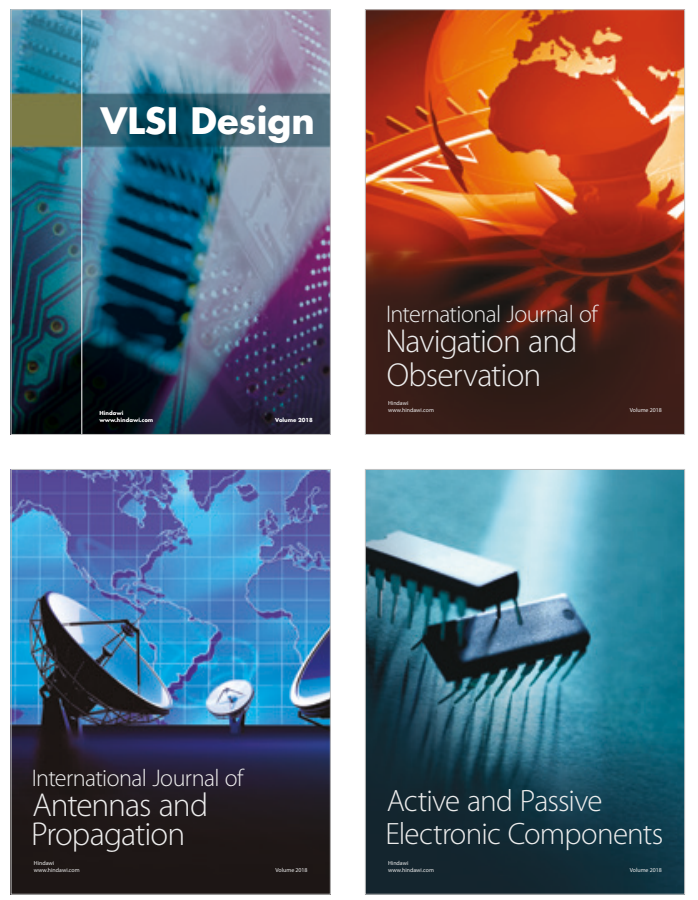
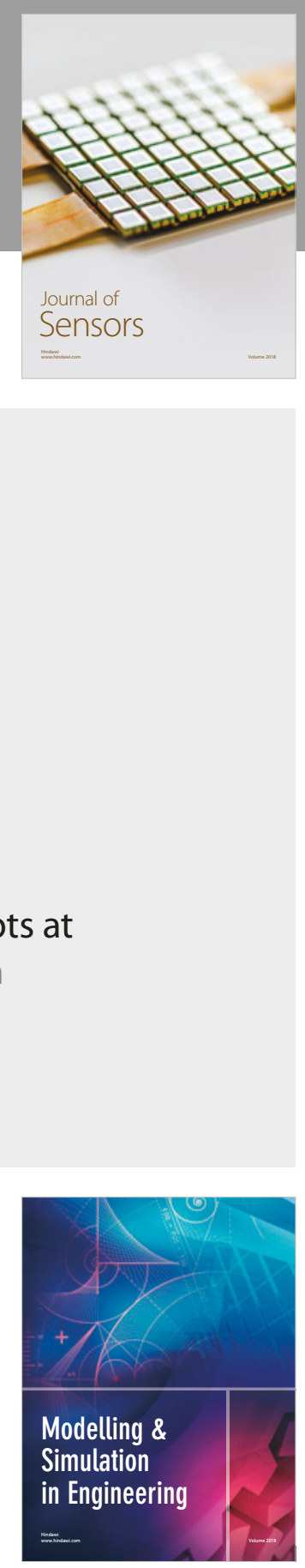

\section{Advances \\ Multimedia}
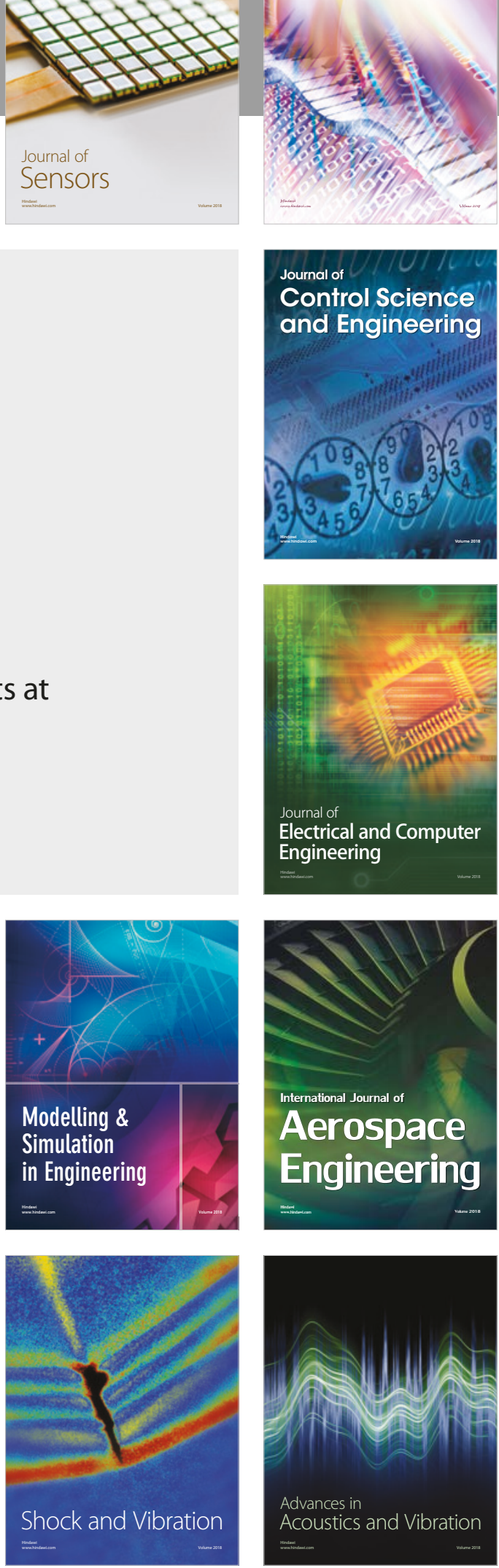